\title{
De mythes en réalités : relations interethniques et questions identitaires en Australie
}

\section{Viviane Fayaud}

\section{(2) OpenEdition}

12 Journals

\section{Édition électronique}

URL : https://journals.openedition.org/jso/5992

DOI : $10.4000 /$ jso.5992

ISSN : $1760-7256$

\section{Éditeur}

Société des océanistes

\section{Édition imprimée}

Date de publication : 15 décembre 2009

Pagination : 195-201

ISBN : 978-2-85430-026-0

ISSN : 0300-953x

\section{Référence électronique}

Viviane Fayaud, «De mythes en réalités : relations interethniques et questions identitaires en Australie », Journal de la Société des Océanistes [En ligne], 129 | juillet-décembre 2009, mis en ligne le 15 décembre 2009, consulté le 21 septembre 2021. URL : http://journals.openedition.org/jso/5992 ; DOI : https://doi.org/10.4000/jso.5992

\section{(c) $(1) \odot$}

Journal de la société des océanistes est mis à disposition selon les termes de la Licence Creative Commons Attribution - Pas d'Utilisation Commerciale - Pas de Modification 4.0 International. 


\section{De mythes en réalités : relations interethniques et questions identitaires en Australie}

par

Viviane FAYAUD*

Le Réseau Asie-Imasie, dont l'objectif est de promouvoir la rencontre des chercheurs travaillant sur l'Asie et le Pacifique, en partenariat avec l'AILAE', a co-organisé du 21 janvier au 12 février 2008 une exposition ${ }^{2}$ réunissant des œuvres d'artistes australiens et des photographies réalisées dans la région de Kimberley ces vingt dernières années par l'anthropologue Kevin Shaw. Regards croisés sur l'identité, tel était le propos de l'ensemble qui a servi de point de départ à une réflexion sur les jeux de représentations, d'une part, entre l'Australie et l'Europe et, d'autre part, sur et entre les communautés aborigènes $^{3}$ ou non aborigènes d'Australie, ouvrant ainsi sur la question de la pluriethnicité, en particulier dans la construction nationale.

Cette réflexion a été menée le 5 février 2008 au sein d'une journée d'étude qui s'est tenue à la Maison des sciences de l'homme (voir le programme complet en ligne http://www.reseauasie.com ${ }^{4}$ ). Intitulée Australie mythes et réalités, elle réunissait selon une approche pluridisciplinaire sociolinguiste, politiste, géographe, anthropologue, spécialistes des études littéraires et historiens, auxquels se sont joints des artistes (cinéaste et écrivain). Ils étaient issus de centres de recherche australiens, britanniques, italiens et français et d'une variété de formations professionnelles et académiques. Chercheurs expérimentés, nantis d'une expérience internationale, chercheurs en début de carrière et doctorants ont confronté leurs recherches sur les imaginaires et les réalités identitaires de l'Australie ${\mathrm{du} \mathrm{XIX}^{\mathrm{e}}}^{\mathrm{e}}$ au $\mathrm{XXI}^{\mathrm{e}}$ siècle. Ne sont publiés ici que cinq des contributions de cette journée, choisies pour leur caractère inédit ainsi que pour l'originalité de leurs corpus, à savoir la littérature et l'art interrogeant les mythes et la réalité ethnique et interethnique australienne, pierre de touche identitaire et enjeu de l'avenir.

\section{Contexte et singularité}

Dès le XVIII ${ }^{\mathrm{e}}$ siècle, la curiosité et l'avidité des Européens ont convergé sur l'Australie, son immensité, ses richesses naturelles et son statut

1. Académie itinérante des échanges Arts et Langues européennes, www.ailae.org.

2. Summo-O'Connell a réalisé le catalogue de cette exposition (Summo-O'Connell, $2008: 1$ ).

3. Précisons que les Aborigènes concernés sont surtout des urbains ou semi-urbains; ils se trouvent donc plus à proximité d'autres migrants que ceux des communautés éloignées.

4. NDLR. - Cette journée comptait quinze communications regroupées en thèmes : littératures (Christine Mathieu, «Goodbye from Vanilla »; Katherine E. Russo et Cyrille Lebourg-Thieullent, dans ce numéro) ; enjeux identitaires (Isabelle Benigno, dans ce numéro; Luc Vacher, "Les espaces du mythe du bush australien : de l'invention du territoire à l'exploitation touristique »); questions aborigènes (Maryvonne Nedeljkovic, «Le paysage dans la pensée aborigène australienne comme extériorité et intériorité »; Géraldine Leroux, « La parole des ancêtres dans les communautés urbaines du Pacifique ») ; mythes et réalités politiques (Anna Cole, "'Dancing with the Prime Minister'. Myth and History »; Fabrice Argounès, " Mythes réalités de la politique étrangère australienne »); art et exil, art de l'exil (Vanessa Castejon, "The exoticism of the Musée du Quai Branly: French perspective on Aboriginal Australia »; Stéphane Sawas, dans ce numéro ; Laetitia Bourget, " Se faire des amis ») ; une introduction (Viviane Fayaud, dans ce numéro ; Renata Summo O'Connell, « Transformation and Art: ethnic and indigenous women artists in Australia ») et une conclusion par Robert Aldrich.

* Réseau Asie-Imasie, uPs 2999 (CNRS-FMSH), vfayaud@msh-paris.fr 
de pays nouveau. Au siècle suivant s'y ajoutent leurs rêves et leurs aspirations à un projet neuf de civilisation s'épanouissant dans un pays enfin équitable (Lagayette, 2008: 7 ; Vernay, 2009 : 45). Aujourd'hui, certains déplorent que l'Australie contemporaine soit un champ et un objet d'investigation périphérique de la recherche française suscitant peu de travaux majeurs, tant il est vrai que chaque pays européen a tendance à travailler sur ses anciens espaces de domination (Royer, 2008 : 135). Toutefois, une certaine fascination semble perdurer et s'est accentuée ces dernières années. Ce n'est pas parce que cet antipode de la France conjugue, à des traditions millénaires des peuples autochtones et à la présence sulfureuse de bagnards et de colons, des aspirations toujours présentes à l'évasion et à la justice sociale que, selon Françoise Kral :

« les études australiennes [...] attirent de plus en plus de chercheurs dans des disciplines aussi différentes que l'histoire, la littérature, la linguistique ou l'ethnologie ». (Kral, $2005: 5)$

Ce renouveau proviendrait plutôt, selon elle, de :

«l'émergence de nouvelles données telles que l'affirmation d'une littérature et d'un cinéma [...] ou encore le débat de fond sur le multiculturalisme, qui intéresse d'autres pays confrontés à des problématiques semblables. » (Kral, $2005: 5)$

D'aucuns soulignent que, d'une manière générale, sur les Aborigènes et le regard occidental eurocentré existent de très nombreuses études. Ainsi, en ce qui concerne le regard de l'Europe sur les Aborigènes, cette littérature de recherche explore notamment les imaginaires que suscite la découverte des peuples autochtones par les explorateurs des $\mathrm{XVIII}^{\mathrm{e}}$ et $\mathrm{XIX}^{\mathrm{e}}$ siècles. Par ailleurs, en anthropologie seulement, les représentations cette fois-ci euro-australiennes des Aborigènes sont le sujet d'une vaste littérature et de nombreux débats. Aussi, nos relecteurs ${ }^{5}$ soulignent, qu'en Australie, le champ des études aborigènes constitue une thématique à part, distincte de l'étude des autres groupes et communautés ethniques. Le dossier se propose une autre approche $^{6}$. Nombre de sociétés contemporaines du
Pacifique, remodelées par les besoins des empires coloniaux, notamment en main-d'œuvre, résultent de multiples mouvements migratoires toujours en cours, vers les pôles de développement économique et, particulièrement, vers la Nouvelle-Zélande ou l'Australie, avec pour conséquences des cristallisations d'identités ethnoculturelles et des revendications politiques.

Ce contexte, allié à la volonté du Réseau AsieImasie d'être fidèle à sa politique de subsidiarité$^{7}$, expliquent les choix de ce dossier qui ne développe pas une anthropologie des Aborigènes mais se focalise sur la mise en place, l'évolution ou la pérennité de l'Australie « à engendrer du rêve » et à forger du symbole affrontant ou négociant :

« [les] dures réalités de la construction d'une communauté nationale multiethnique et multiculturelle. » (Lagayette, $2008: 8$ )

Dans le cadre de cette problématique, les Aborigènes sont associés plutôt que particulièrement distingués des autres communautés d'Australie. La fascination pour l'Australie, issue ou non de la nation, face aux réalités des relations entre communautés, perçues et imposées de l'extérieur par l'altérité ou dominées par la construction de l'image de soi, permet également, sans se désintéresser des pratiques et des aspects des périodes coloniale et post-coloniale, de se placer au-delà de leurs problématiques ${ }^{8}$. L'éviction d'une vision binaire de l'Australie isolant les autochtones colonisés (ou Aborigènes) des colonisateurs et des migrants, éviction qui est loin d'être originale puisqu'elle existe depuis plusieurs décennies, autorise également une appréhension différente, peut-être plus fine, des multiples positions médianes, similarités et/ou différences nées d'interactions et d'échanges continus, alors que par la bipolarisation, les autres :

" sont toujours présentés comme un collectif homogène appelé "ils", collectif qui s'oppose à un autre collectif, "nous" » (Russell, $2006: 2$ )

chaque entité étant dotée de caractéristiques généralement peu nuancées. En outre, celui qui parle se place au centre repoussant souvent les

5. NDLR. - L'auteur fait référence ici aux avis de lecture transmis par la rédaction du JSO aux auteurs des articles.

6. Cette situation entraînerait « une plus grande amnésie concernant d'autres histoires y compris les histoires des migrations d'après-guerre » (Brewster, 1995 : 17, citant Gunew et al., 1993 : 449, traduit par nous).

7. Le réseau Asie-Imasie promeut des recherches selon des démarches et/ou dans des domaines différents de ceux que les laboratoires de recherche et les institutions existantes poursuivent, se voulant en complémentarité et non en concurrence de leurs travaux.

8. Par les politiques du multiculturalisme et de la Réconciliation nationale, l'Australie se perçoit comme une nation post-coloniale (Lagayette, 2008 : 12). Cela ne signifie pas que la réconciliation soit effective. Pour faire simple, post-colonial est ici surtout pris dans son sens historique (Brewster, 1995 : 22). La perspective post-moderne s'interroge sur les résultats de l'anthropologie ou le concept de vérité historique, chacune impliquant des choix et des points de vue spécifiques qui les placeraient surtout dans le domaine du discours. 
autres à la marge. De plus, les « Aborigènes » eux-mêmes sont loin de constituer une entité homogène et uniforme (Russell, 2006 : 4). Enfin, ces constructions ne prennent pas toujours en compte le dynamisme des cultures qui, loin d'être figées et immuables, évoluent (Brewster, 1995 : 13). Manifester les positions médianes et les nuances au sein d'un groupe en apparence homogène qu'il s'agisse des Français explorateurs de l'Australie au XIX ${ }^{\mathrm{e}}$ siècle (Fayaud), des écrivains euro-australiens, colons anglais ou migrants grecs (Benigno et Sawas) s'avère plus aisé si l'objet d'étude bénéficie d'une contextualisation historique et/ou d'une attention aux parcours personnels. On remarque alors les positions divergentes qu'inspirent les autochtones aux savants français en exploration, ou l'hétérogénéité de l'hellénisme australien ${ }^{9}$. Une commune expérience explique également la réunion dans un même dossier de sujets d'études traditionnellement pris séparément, tels les Aborigènes et les migrants grecs, car, selon Simone Rinzler, migrants et déracinés partagent avec ceux qui ont subi la colonisation, l'exil ou l'exode, le besoin de reconnaissance, une demande de considération qui conduit à la proclamation de l'identité et à l'expression dans l'espace public de l'expérience du déracinement, du choc des cultures, de la discrimination et des préoccupations concernant la langue ou l'identité hybride (Rinzler, 2005 : 179-180 ; Vernay, 2009 : 118). Cette approche, nombre d'Aborigènes eux-mêmes l'acceptent, notamment sur le thème de la discrimination (Pons, 2008 : 115). Toutefois, les articles de ce dossier, qui reflètent tout à la fois l'état actuel de la recherche et la polarisation politique et culturelle, portent largement leur attention au monde aborigène.

Présenter des travaux de recherche majoritairement francophones sur l'Australie contemporaine $\left(\mathrm{XIX}^{\mathrm{e}}\right.$ au $\mathrm{Xx}^{\mathrm{e}}$ siècle) et ne pas distinguer particulièrement une communauté ethnoculturelle ${ }^{10}$ pour appréhender les nuances et les variations dans les perceptions et les expressions constituent les deux premiers aspects de ce dossier. Le troisième réside dans les corpus utilisés car il s'agit de sources recevant une considération récente pour la compréhension des questions interethniques et identitaires, à savoir l'art (Russo) et la littérature (Benigno et Sawas). D'autres sources ne sont que rarement voire jamais consultées : l'art de l'illustration (Fayaud et Lebourg) ou la littérature de jeunesse (Lebourg). Enfin, le dossier prend en compte la perception selon le genre, puisqu'une artiste aborigène et trois écrivaines euro-australiennes (anglaise et grecques) sont passées au crible de l'analyse. Il évoque la confrontation des centres (ouvrages scientifiques et presse de Paris, littérature de jeunesse de Londres) aux périphéries (la littérature des migrants grecs, l'art aborigène), mais inversement des périphéries devenant centres (la littérature des Euro-australiens " angloceltes ») et des paradoxes qui surgissent entre leurs principes et leurs entreprises.

\section{Dépeindre : appréhension extérieure et compréhension}

Dans ces sociétés en mutation, les productions artistiques peuvent jouer un rôle capital. Les formes artistiques des peuples premiers de l'Australie ont donné lieu à une vaste bibliographie, qu'il s'agisse de leur littérature ou de leurs œuvres picturales. Peut-on mettre en évidence l'apport original des productions esthétiques afin de mieux cerner leurs fonctions sociales ? Les travaux en la matière, mais ils ne sont pas encore très nombreux, démontrent peut-être surtout à quel point les théories des productions symboliques sont le reflet des sociétés de type occidental. Pourtant, un historien souligne que :

«jamais peut-être la reconnaissance d'un peuple n'est autant passé par son art que pour les Aborigènes. » (Barbe, 2009 : 616)

Cependant, rappelle Xavier Pons, les artistes aborigènes travaillent avec des mediums et des supports modernes. Ils usent de l'acrylique et non des pigments minéraux ou végétaux, et de la toile ou du carton au lieu de l'écorce. Surtout, les thèmes se sont transformés : spiritualité chrétienne ou militantisme politique (Pons, 2008 : 105). Cette évolution est-elle une perte d'authenticité ? Katherine Russo, de l'université de Naples, analyse les peintures d'une artiste aborigène contemporaine en regard des concepts d'authenticité, d'hybridité et d'intersubjectivité, en prenant appui sur des travaux récents de type anthropologique en Australie et surtout sur ceux d'Homi Bhabba (1994). Prisme tout aussi original, l'art de l'illustration (Fayaud et Lebourg), art mineur à l'impact non négligeable, est ici

9. «L'hellénisme est un phénomène à la fois démographique, culturel et sociopolitique [...]. On entend par ce terme l'ensemble des populations qui se revendique ou se sont revendiquées dans un passé plus ou moins lointain d'une identité grecque » (Bruneau, $2000: 35$ ).

10. Même si une expérience commune de la marginalisation ne débouche pas sur des positions communes. Les Aborigènes refusent d'être concernés par la politique du multiculturalisme. 
largement sollicité. Les représentations iconographiques cristallisent les intérêts, les activités, les conceptions et les sentiments du centre, fondements et reflets de la vision sur la périphérie mais reflets également de sa manière de la traiter. Comprendre les enjeux de la représentation exige une contextualisation historique, encore absente pour les dessins des navigations françaises. En effet, l'iconographie coloniale du $\mathrm{XIX}^{\mathrm{e}}$ siècle ${ }^{11}$ n'est que très rarement remise dans une perspective historique, et encore moins replacée dans l'évolution du milieu artistique. Innovateur également l'angle considéré, à savoir ce qui modèle et influence l'iconographie de l'Australie, pays qui pour l'art représente avant tout une source de motifs, et non la représentation du « bon » ou " noble » et de « l'ignoble » sauvage, objet de nombreuses analyses critiques (Hartog, 2005 ; Ellingson, 2001 ; Dickason, 1995 ; Vibart, 1984). L'implantation étrangère et la découverte de l'or ont été, entre autres, porteuses d'un imaginaire qu'illustrent tant la presse française que les intrigues de romans ${ }^{12}$. Par elles l'Australie entre dans la littérature française, qui, à l'image de son homologue anglaise :

«s'adresse prioritairement au jeune garçon occidental, lui offre l'occasion d'é/prouver sa virilité et de tester ses qualités d'homme blanc. » (Ryan-Fazilleau et al., 2008: 69)

L'analyse intéressante et surtout novatrice de Cyrielle Lebourg se focalise sur les représentations iconographiques de cette littérature de jeunesse (1830-1930). L'historien Alain Corbin a clairement démontré qu'il n'est plus possible de :

« disqualifier la littérature romanesque sous prétexte qu'elle relève de l'imaginaire et ne renvoie qu'à elle-même. » (Corbin, 1986 : 85)

Depuis Edward Saïd, les études culturelles explorent la portée sociopolitique et géopolitique des réalisations culturelles ${ }^{13}$ (Saïd, 2000 : 28). Jacques Rancière parle même de Politique de la littérature qui :

« suppose qu'il y a un lien essentiel entre la politique comme forme spécifique de la pratique collective et la littérature comme pratique définie de l'art d'écrire. » (Rancière, 2007 : 11)

La littérature australienne, de son origine aux années 1920 :
« se veut interprète de la réalité telle que la voient ou la souhaiteraient les colons.» (Nedeljkovic, 1982:15)

Elle témoigne précisément des interactions sociales (l'identité, la classe, le genre, les minorités, la marginalité, le multiculturalisme, le migrant), ethniques (la race, l'étranger, Noirs ou Blancs, l'ethnicité), et des questions contemporaines (l'exil, le nationalisme ou comment bâtir/caractériser la nation). Ayant rejeté le formalisme et l'intellectualisme occidental dans son expression du monde et de son sens, elle s'est constituée laboratoire des évolutions symboliques, des conflits et de l'avenir.

L'enjeu de l'avenir constitue, dès la fin du $\mathrm{XVIII}$ e siècle, un élément clé de la richesse symbolique de l'Australie. Le pays génère la foi en l'avenir. En France, elle relève très tôt de préoccupations utopiques, l'Australie représentant un antipode social où il est possible de bâtir une meilleure société sans classes, récompensant socialement et financièrement selon le mérite et non selon la naissance. Cette vision de l'Australie comme monde meilleur persiste puisque :

«dans la littérature française depuis les années 1960, [...] les indigènes australiens sont dépeints comme le dernier rempart contre les ravages de la modernisation. » (Ryan-Fazilleau et al., 2008 : 18)

À l'interne, cette confiance ressort des « premiers écrits littéraires », qui «parlent en terme d'avenir et non de passé », qu'une minorité d'auteurs conçoivent en rupture avec l'Angleterre considérée comme "désuète, austère et lointaine », mais aussi comme synonyme de conservatisme et d'injustice sociale (Nedeljkovic, 1982 : 11). Pour l'Australie revendicatrice de son identité, il s'agit

« [d']élaborer à partir de l'expérience passée l'éthique future d'une nation et d'une civilisation naissantes. » (Nedeljkovic, $1982: 16)^{14}$

Cependant, cette foi en l'avenir croît au sein de l'épineuse question des relations interethniques. Le mythe de l'homogénéité nationale butte sur la réalité du peuplement multiethnique et multiculturel (Piquet, 2004 : 36). Des romanciers mettent en lumière non l'héroïsme entêté des Australiens blancs mais leur conduite d'envahisseurs destructifs, et élèvent leurs voix contre les discrimi-

11. Pour le XVIII ${ }^{\mathrm{e}}$ siècle en général et pour le $\mathrm{XIX}^{\mathrm{e}}$ siècle anglo-saxon, voir les analyses inégalées de Bernard Smith (1985).

12. Une cinquantaine de romans entre 1852 et 1910, la plupart dans le sillage de Jules Verne (Ryan-Fazilleau et al., $2008: 67$ et 80 ).

13. «Des réalignements inédits s'opèrent, rapidement, à travers frontières, types, nations et essences, et ce sont eux désormais qui défient la notion fondamentalement statique d'identité, cœur de la pensée culturelle à l'ère de l'impérialisme » (Saïd, $2000: 28$ )

14. Voir également Pons (2005: 59). 
nations et injustices touchant les Aborigènes. Aussi la majorité des études sur la littérature australienne porte-t-elle sur la représentation des Aborigènes, du bush ou de la femme. Isabelle Benigno analysant Coonardoo de Katharine Susannah Prichard (1883-1969) sacrifie à ces thématiques puisque les femmes pionnières et les relations raciales sont le centre des sagas historiques de la romancière qui menait, par ailleurs, un combat en faveur des Aborigènes donnant ainsi à ses œuvres une dimension politique. Première à accorder aux Aborigènes un rôle majeur dans la littérature, Katharine S. Prichard les présentent en sauveurs bienveillants ou initiateurs de l'homme blanc à la terre australienne. Ses ouvrages dévoilent également le fossé entre des principes avoués d'égalité et des pratiques sous-jacentes, où dans Coonardoo par exemple, l'attraction homme-femme ne peut s'épanouir que dans le respect des frontières rigides de la division raciale, conduisant dans le cas inverse les protagonistes à l'impasse, ballottés de l'incompréhension mutuelle à la destruction (Bennett et al., 1998 : 102, 112, 123, 210).

À côté des femmes et des Aborigènes, les migrants sont souvent des protagonistes problématiques en raison même de leur marginalisation avec les cultures dominantes et des négociations qu'exigent d'eux les méfiances de leurs compatriotes. Stéphane Sawas analyse la littérature de la communauté périphérique des migrants grecs qui reflète toute l'originalité de l'Australie pluriethnique et plurilingue, où le grec est largement parlé. La découverte de l'or vers 1850 accélère et diversifie l'implantation étrangère sur le sol australien. Une communauté grecque prend corps (MacLeod, 2006:111 et 123). À la fin du siècle, Melbourne, où s'érigeait dès la fin $\mathrm{du} \mathrm{XIX}^{\mathrm{e}}$ siècle la première église grecque orthodoxe d'Australie, et Sydney rassemblaient environ un millier d'immigrants grecs. Malgré son ancienneté, cette diaspora n'était pas très estimée et était vouée à végéter au bas de l'échelle socioprofessionnelle puisque les carrières prestigieuses et rémunératrices lui était refusées. «Ses ressortissants, jugés préférables aux immigrés de couleur », rappelle Xavier Pons, étaient néanmoins considérés comme « race » notoirement inférieure (Pons, 1996 : 43 ; Piquet, 2004 : 95). Ils subirent à plusieurs reprises des pillages et des exactions de la part des mineurs d'origine anglosaxonne. En effet, jusque vers 1950, l'Australie a rejeté avec obstination toute intégration hors des «Anglo-Celtes » (Pons, $1996:$ 42-3, 70, 211 ; Piquet, 2004 : 11, 95).

« Le sentiment de rejet et la volonté d'expulsion que manifestaient les colons britanniques ne s'appli- quaient pas uniquement aux communautés de couleur, mais à tout groupe suffisamment nombreux pour paraître menacer l'homogénéité raciale des colonies. " (Piquet, 2004 : 95)

S'accroissant après la migration de masse de 1947, la communauté grecque représente aujourd'hui $4 \%$ de la population australienne ; Melbourne est «l'une des plus grandes villes grecques du monde » (Pons, 1996 : 334) et c'est à leur intention (et celle des Italiens) que le multiculturalisme est instauré à partir de 1973 (Bruneau, $2000: 35$ ). La politique du multiculturalisme s'ancre avec force dans la littérature qui cesse d'être l'apanage des "Anglo-Celtes ». Si des études ont été consacrées à la littérature des Italiens, des Allemands, des Chinois d'Australie, celles portant sur les écrivains migrants grecs sont plus rares. Or, ces derniers, souligne l'Oxford Literary History of Australia, ont été les plus importants des écrivains migrants, "pour des raisons qui ne sont pas totalement claires » (Bennett et al., 1998:274). Cette dernière remarque souligne explicitement que des recherches restent à mener. Ce même ouvrage d'histoire littéraire australienne rappelle l'impact de Dimitri Tsaloumas et Antigone Kefala, sans toutefois faire plus que leur reconnaître cette prééminence puisqu'il ne cite qu'une fois en presque cinq cents pages deux des trois auteurs étudiés par Stéphane Sawas (Bennett et al., $1998: 274$ et 326). Le sujet méritait donc un regard plus approfondi. En analysant les diverses expressions littéraires des Grecs d'Australie, Stéphane Sawas parvient à mettre au jour à la fois l'évolution méconnue de la problématique identitaire dans l'Australie des $\mathrm{Xx}^{\mathrm{e}}$ et $\mathrm{XXI}^{\mathrm{e}}$ siècles et l'impact de la gestion politique du multiculturalisme sur les stratégies éditoriales et la diffusion de textes littéraires pas toujours à l'abri de récupérations inattendues.

L'ensemble des contributions dévoile que la question de l'ethnicité ne peut être dissociée de l'identité australienne. Ce dernier thème hante les artistes car l'Australie exacerbe le désir d'appartenance. Katharine S. Prichard par exemple avait donné confiance et corps à l'émergence d'une conscience nationale, se dépensant pour forger un socle de valeurs proprement australiennes, ce qu'atteste l'écrivain Frank Dalby Davison (1893-1970) qui déclarait :

«Vous ne comprendrez jamais quel impact le livre de Katharine, Working Bullocks, a eu sur nous. Voici un livre écrit par un Australien, écrit aussi bien que n'importe lequel des écrivains étrangers, un livre avec un activiste militant comme héros - c'est par ce livre que tout a commencé pour moi 
et Palmer et tous les autres. $\rangle^{15}$ (Bennett et al., $1998: 206)$

Si les études critiques d'histoire nourrissent la construction identitaire nationale, comme le rappelle Lynett Russell aujourd'hui :

« dans le discours australien post-colonial, les études sur l'identité, et les notions de nation, le concept d'hybridité joue un rôle important - si ce n'est central. $\gg^{16}$ (Russell, $2006: 3$ )

À l'issue de ces études, il reste à s'interroger sur la dimension du mythe et de la réalité dans l'idée d'homogénéité socioculturelle comme fondement d'une communauté et d'une nation, concept qui alimente des identités nationales peut-être plus proches elles aussi du mythe que de la réalité. Plus largement demeure la question, soulevée par François Laplantine, de la pertinence de la notion d'identité. L'altérité n'est-elle pas l'une des questions les plus délicates et des plus brûlantes du monde d'aujourd'hui ?

\section{BIBLIOGRAPHIE}

Barbe Dominique, 2009. Histoire du Pacifique des origines à nos jours, Paris, Perrin.

BennetT Bruce and Jennifer Strauss (eds), 1998. The Oxford Literary History of Australia, Oxford, Oxford University Press.

BhabHa Homi, 1994. The location of culture, London and New York, Routledge.

BrewSTER Anne, 1995. Literary formations, postcolonialism, nationalism, globalism, Melbourne, Melbourne University Press.

BRUNEAU Michel, 2000. Hellénisme et diaspora grecque, de la Méditerranée orientale à la dimension mondiale, Cahiers d'étude sur la Méditerranée orientale et le monde turco-iranien 30, pp. 32-56.

Corbin Alain, 1986. Le Miasme et la Jonquille. L'odorat et l'imaginaire social aux $X_{X I I I}{ }^{e}-X I X^{e}$ siècles, Paris, Flammarion.

Dickason Olive Patricia, 1995. Le mythe du sauvage, Paris, P. Lebaud.

Ellingson Terry Jay, 2001. The myth of the noble savage, Berkeley, University of California Press.

Gunew Sneja et Anna Yeatman (eds), 1993. Feminism and the Politics of Difference, Sydney, Allen and Unwin.

Hartog François, 1991. Le miroir d'Hérodote, essai sur la représentation de l'autre, Paris, Gallimard.
—, 2005. Anciens, Modernes, Sauvages, Paris, Galaade Éditions.

KraL Françoise (éd.), 2005. Re-presenting otherness, Mapping the Colonial 'self', Mapping the Indigenous 'other' in the literature of Australia and New Zealand, Nanterre, Publidix-université de Paris-X, Nanterre.

Lagayette Pierre (éd.), 2008. Rencontres australiennes, regards croisés sur l'identité d'un peuple et d'une nation, Paris, Presses universitaires de la Sorbonne.

LAPlantine François, 1999. Je, nous, les autres, Paris, Pommier.

MacLeod Celeste Lipow, 2006. Multiethnic Australia, Jefferson, Mcfarland.

NedeljKovic Maryvonne, 1982. L'aube d'une nation, les écrivains d'Australie de 1788 à 1910, Paris, PUF.

Piquet Martine, 2004. Australie plurielle, gestion de la diversité ethnique en Australie de 1788 à nos jours, Paris, L'Harmattan.

Pons Xavier, 1996. Le multiculturalisme en Australie, au-delà de Babel, Paris, L'Harmattan.

—, 2005. Les mots de l'Australie, Toulouse, Presses universitaires du Mirail.

-, 2008a. Black Janus, Cross-Cultural Strategies in Aboriginal Writing, in Pierre Lagayette (éd.), Rencontres australiennes, regards croisés sur l'identité d'un peuple, d'une nation, Paris, Presses universitaires de la Sorbonne, pp. 103-111.

-, 2008b. Seeing Double: Visions of Australia in two French Crime Novels, in SueRyan-Fazilleau and Serge Linkès (eds), France and Australia Face to Face, Australie/France, regards croisés, Paris, Les Indes Savantes, pp. 81-108.

Rancière Jacques, 2007. Politique de la littérature, Paris, Galilée.

RinzLer Simone, 2005. E Pluribus Unum: the Vindication of a Fictional Aboriginality, Reading Mudrooroo's Us Mob in the Light of Lecercle and Rushdie, in Françoise Kral (éd.), Re-presenting otherness, Mapping the Colonial 'self' Mapping the indigenous 'other' in the literatures of Australia and New Zealand, Nanterre, Publidix-Université Paris-X Nanterre, pp. 171-198.

ROYER Ludivine, 2008. Research on Australia: A voice from abroad, in PierreLagayette (éd.), Rencontres australiennes, Regards croisés sur l'identité d'un peuple et d'une nation, Paris, Presses Universitaires de la Sorbonne, pp. 119-138.

Russell Lynette (ed.), 2006. Boundary writing, an exploration of race, culture, and gender binaries in contemporary Australia, Honolulu, University of Hawai'i Press.

15. «You'll never know what an impact that book of Katharine's, Working Bullocks, had on us. Here was a book written by an Australian, written as well as any of the foreign writers, a book with a militant activist as a hero - this book started it all for me and Palmer and the others. » (Traduit par nous).

16. " [...] hybridity plays an important - if not central - role in Australian postcolonial discourse, identity studies and notions of nationhood. » (Traduit par nous). 
Ryan-Fazilleau Sue et Serge Linkès (eds), 2008. France and Australia, face to face; Australie/France: regards croisés, Paris, Les Indes savantes.

SAÏD Edward W., 2005 (1 ${ }^{\mathrm{e}}$ éd. 1978). L'orientalisme, l'Orient créé par l'Occident, Paris, Seuil.

—, 2000. Culture et impérialisme, Paris, Fayard-Le monde diplomatique.

Smith Bernard, 1985. European Vision and the South Pacific, London, Yale University Press.

Summo-O'Connell Renata et Viviane FAyAud (éds), 2008. Australie, mythes et réalités, cata- logue de l'exposition, Florence, Grafiche la stamperia.

Vernay Jean-François, 2009. Panorama du roman australien des origines à nos jours, 1831-2007, Paris, Hermann éditions.

VIBART Éric, 1984. Aspects du mythe du « Bon sauvage » océanien à travers les récits et la littérature de voyage du dix-huitième siècle, thèse de Littérature, Paris IV.

WoLfe Patrick, 1999. Settler colonialism and the transformation of anthropology, London, Cassell. 
